\title{
Transcriptome Profiling of Elaeis guineensis Jacq. Under Heat Stress Condition
}

\author{
Sigit Dwi Maryanto ${ }^{\text {a, }}$, Roberdi Roberdi ${ }^{a}$, Zulfikar Achmad Tanjung a, Wulan Artutiningsih ${ }^{\text {a }}$, \\ Olivia Sriulina Purba ${ }^{a}$, Tengku Imam Saputra ${ }^{a}$, Condro Utomo ${ }^{a}$, Tony Liwang ${ }^{\text {a }}$ \\ ${ }^{a}$ Biotechnology Department, Plant Production and Biotechnology Division, PT SMART Tbk., Babakan Madang, Bogor 16810, \\ West Java, Indonesia \\ Corresponding author: *biotechnology@sinarmas-agri.com
}

\begin{abstract}
Global warming is predicted to have a generally negative effect on agriculture activity. High temperatures stress could affect plant growth negatively. Developing plants with improved thermal tolerance using molecular genetic approaches could mitigate these heat stress effects. Elite palms with better adaptation to heat can be selected from germplasm using molecular markers. Transcriptome profiling by RNA sequencing is a way to find molecular markers of a particular trait. The objective of the study was to obtain differential expressed genes (DEGs) related to the heat stress effect. RNA sequencing results were displayed using heat maps which were useful for visualizing the expression of genes across the high-temperature treatment and control samples. In total, where 1,087 genes were identified involved in oil palm heat stress. Sixty-four (64) of them were differentially expressed, consisted of seventeen (17) up-regulated and forty-seven (47) down-regulated. The uni-gene was summarized in Gene Ontology (GO) categories, namely: biological process, molecular function, and cellular component, subsequently divided into 53 sub-categories. The single organism process, biosynthetic process, response to stimulus, oxidation-reduction process, and response stress were the five primary sub-categories. Sixty-four genes related to heat stress were found, and eight $(12.5 \%)$ of them were determined as heat shock protein (HSP) family. The highest transcription level was the uncharacterized gene, a member of the heat response sub-category, and the others up-regulated gene consisted of HSP family gene, Bcl-2-associated athanogene (BAG) family and HIPP gene, slr0575 gene, CML14 gene, and PARP gene.
\end{abstract}

Keywords — Differentially expressed genes; heat shock proteins; heat-temperature; oil palm; RNA-sequencing.

\section{INTRODUCTION}

Global temperature has increased by about $0.74^{\circ} \mathrm{C} \pm 0.18^{\circ} \mathrm{C}$ in the last 100 years on average [1]. Some Indonesian oil palm plantations have a temperature range above the optimum temperature for oil palm. The optimum mean range temperature for oil palm growth is $24-28{ }^{\circ} \mathrm{C}$, while the minimum temperature is $18{ }^{\circ} \mathrm{C}$ and the maximum temperature is $36{ }^{\circ} \mathrm{C}[2]$. Heat, nutrient deficiencies, drought, cold, and salinity, are the major abiotic stresses faced by plants. These stresses are often connected, combination or individually. The stress will affect plant growth by changing biochemical, physiological, and morphological changes that adversely affect yield [3]. Moreover, global warming has a negative effect on planting growth and development. There is a combined function of time, degree of increase in temperature, and the intensity of temperature in degrees [4].
The molecular mechanism of abiotic stress is predicted control and triggering of specific stress-related genes [7]. The genes participate in transcriptional control, free radical and toxic compound scavenging, signaling, membrane, and protein protection due to stress. Heat shock proteins (HSPs) are genes induced by heat stress. HSPs have a function as molecular chaperones [8]. Induced HSP in tomato heattolerant genotypes was higher compared to susceptible ones [5]. Heat stress reduces plant photosynthetic and respiratory activity, leading to a shortened life cycle and diminished plant productivity. High temperature can modify starch accumulation, sucrose synthesis, and carbon metabolism enzymes [5]. The initial effects of temperature stress comprise decreased enzymes activity and structural change of protein complexes of chloroplast [6]. Membrane permeability which may alter elongation, differentiation, and extension of cells, might change by heat stress.

Transcriptome profiling of heat stress treatment was analyzed in Arabidopsis thaliana, maize, and wheat [7]. 
Transcriptome profiling analysis showed that the metabolism and development-related genes are more highly expressed in tomato heat-sensitive genotypes and associated with a lower percentage of transcripts affected by heat-tolerant progeny [8]. To date, there is no report of the transcriptome profiling of oil palm treated with heat stress. Therefore, the objective of this study was to obtain the differential expressed genes (DEGs) genes related to heat stress in oil palm. This study provided insight into the molecular mechanisms of the heat stress response in oil palm and may be useful in breeding programs.

\section{MATERIALS AND MethodS}

\section{A. Plant Materials and Field Trials}

Four months old of four seedlings of two oil palm progenies were treated with high temperature and control. Planting materials were Tenera (DxP) consisted of Progeny A and Progeny B as a control oil palms seedling were grown in a greenhouse under a controlled temperature condition of 28 $-30{ }^{0} \mathrm{C}$. Heat stress was applied to the treatment samples by raising the modified growth chamber temperature to $36-45$ ${ }^{0} \mathrm{C}$ for 6 hours per day for 14 days. Leaf and root samples were collected at 7 and 14 days after treatment (DAT) according to Rankine and Fairhurst method's [9] and put in RNA Later (Thermo Scientific, Massachusetts, USA) immediately to preserve the RNA.

\section{B. RNA Isolation and RNA Sequencing}

According to the manufacturer's instruction, total RNA was extracted from the leaf and root sample by Rneasy Plant Mini Kit (QIAgen, Hilden, Germany). Extracted total RNA was measured using NanoDropTM 2000c Spectrophotometer (Thermo Scientific, Massachusetts, USA). Good quality RNA was preserved by RNAstable (Biomatrica, California, USA). These RNAs were shipped to Novogene Co., Ltd., Beijing, China [10].

The RNA quality was checked using the 2100 Bioanalyzer Instrument (Agilent Company, USA). Electrophoresis was used as a quality control procedure of biomolecules. The RNA parameters were calculated, including RNA concentration, rRNA ratio, and RNA integrity number (minimum 6.0). RNA was converted into cDNA, and sequencing adapters were added. The cDNA was sequenced using the Illumina platform [12].

\section{Gene Expression Analysis}

Gene expression analysis was conducted by the RT-qPCR method (Applied Biosystems Fast 7500/7500 machine, USA) using Quantifast SYBR Green PCR Kit (Qiagen, Hilden, Germany) a fluorescent signal. RT-qPCR was applied for the uncharacterized LOC105045046 gene, the highest fold change DEG in RNAseq data.

Primer was designed using Primer 3 online software (https://www.bioinformatics.nl/cgibin/primer3plus/primer3pl us.cgi). Gene expressions were analyzed in root and leaf samples using relative quantification [11]. Cq and quantity value of the sample were obtained automatically from the qPCR analysis software v2.0.6 7500, while Relative Quantitation (RQ) value was calculated using a formula based on Applied Biosystems guidance [12].

\section{Data Analysis}

The Kallisto software was used as a raw count of RNA quantification [13]. Transcript per million (TPM) was used as expression normalization. DESeq2 was used for differential expressed gene analysis. Plant transcriptional regulatory map database was used for gene ontology enrichment analysis [14]. Microsoft Excel analyzed the annotation of DEGs with terms describing biological processes, molecular functions, and cellular components.

\section{RESULTS AND DISCUSSION}

\section{A. Transcriptome Profile}

RNA sequencing results were commonly displayed using heat maps useful for visualizing gene expression in treatment and control samples [10]. The number of identified genes was 1,087 DEGs. Heat-map showed that genes were expressed differently in particular treatment of both progenies (Figure 1). Overall, the heat map has shown a contrasting profile between treatment and control on $7^{\text {th }}$ and $14^{\text {th }}$ DAT in both progenies. Seventeen genes were upregulated, and 67 genes were down-regulated in heat-treated samples. Progeny A showed a different transcriptome profile with progeny B. Progeny B showed higher fold change in some genes than Progeny A both at $7^{\text {th }}$ and $14^{\text {th }}$ DAT.

Plants have different mechanisms for surviving under heat stress. The plants' adaptation systems consisted of transpiration cooling, alteration of membrane lipid compositions, and changing leaf orientation in the short-term during the stress period. The early stress signals would trigger downstream transcription controls and plant signaling processes. They would activate the stress-responsive mechanisms to repair the proteins and membranes damages, protect, and redevise homeostasis [15]. Insufficient responses at several steps within the signaling and gene activation may ultimately result in permanent damages in destruction of functional and cellular homeostasis, leading to cell death, and structural proteins and membranes [16]. 


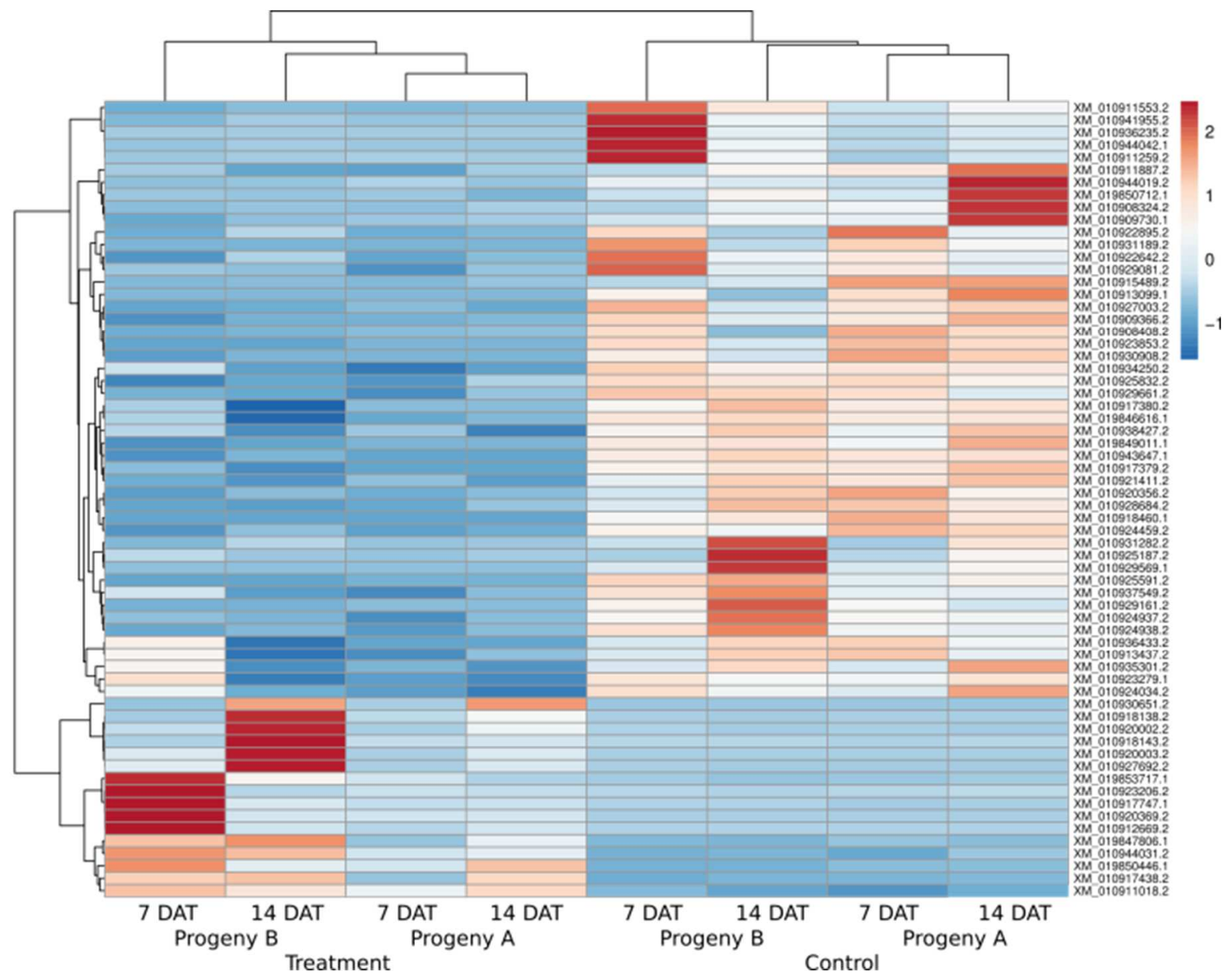

Fig. 1 Heat map of transcriptome profile heat stress vs control

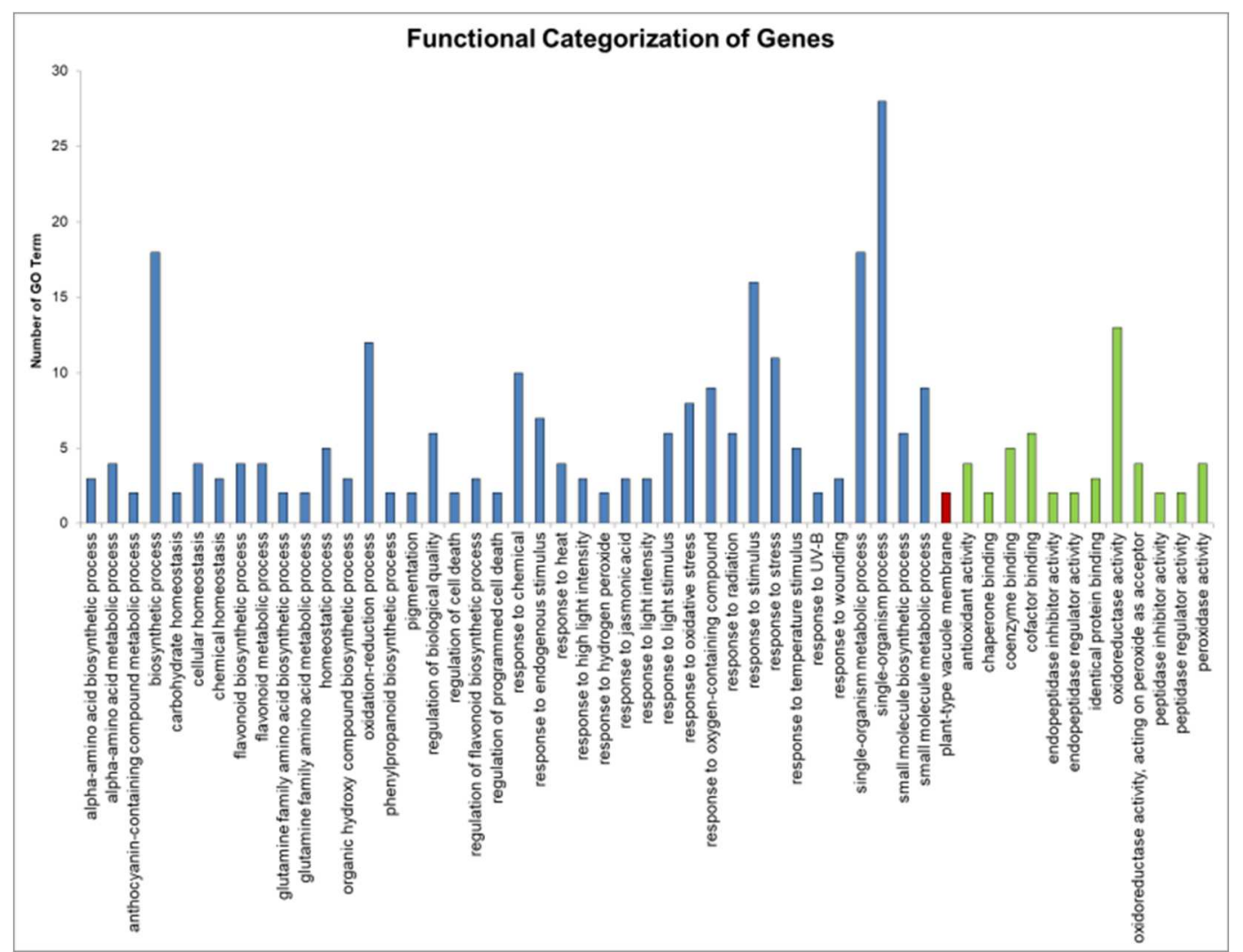

Fig. 2 Functional categorization of genes related heat stress in oil palm, blue color: biological process; red color: cell component; green color: molecular function 


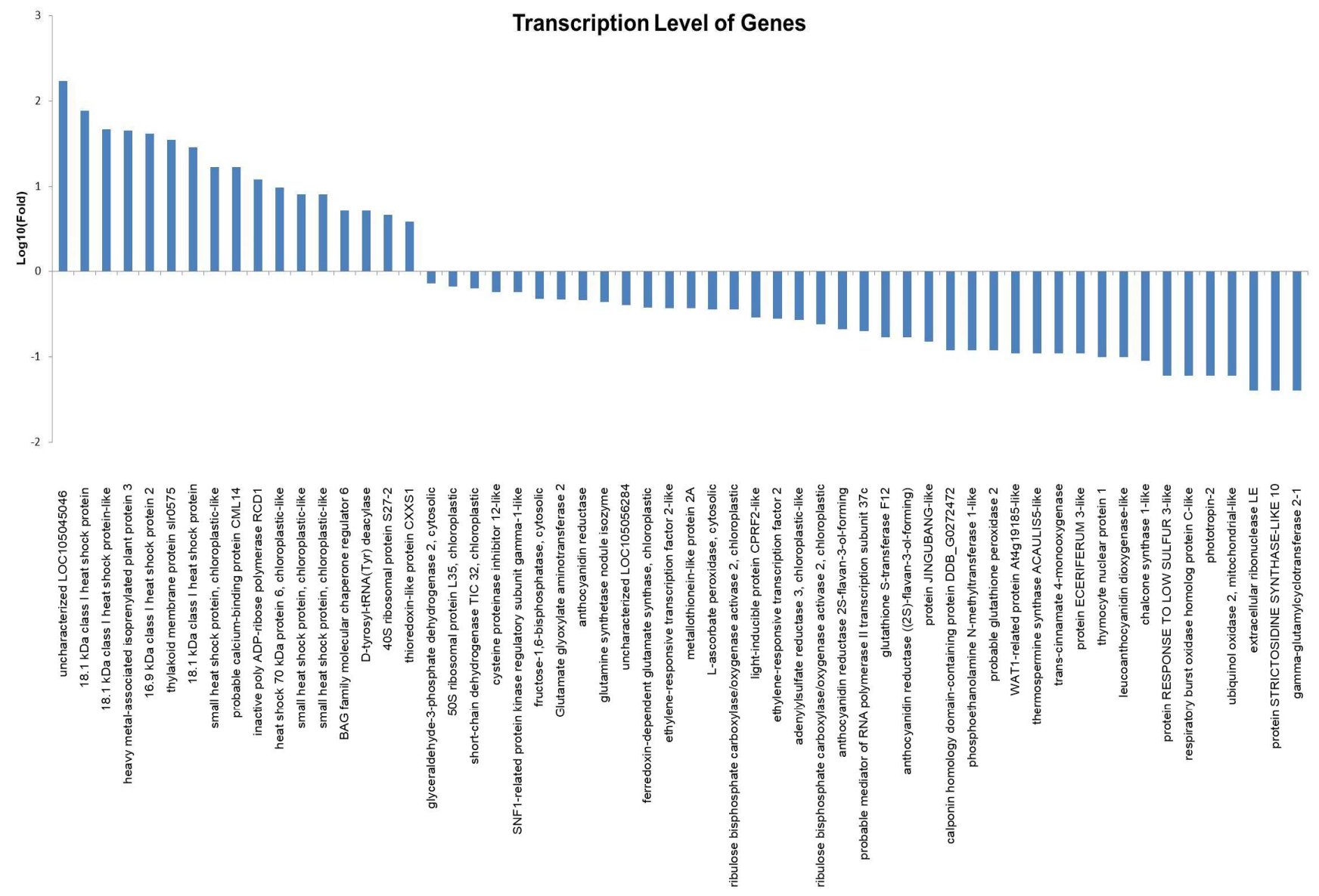

Fig. 3 Transcription level of genes responding to heat stress

\section{B. Gene Ontology}

Functional genes related to heat stress were summarized in three main GO categories (biological process, cellular component, and molecular function) [18] and divided into 53 sub-categories (Figure 2). The biological process consisted of 40 sub-categories. The top-five class consisted of a single organism process, biosynthetic process, response to stimulus, oxidation-reduction process, and response stress response. The response stress process sub-category is clustered genes that are regulated under stress [19]. The stimulus-response of an organism is indicated as a process that causes changes in cells activities, secretion, gene expression, enzyme production, and cellular responses.

Plants reacted to heat stress through synthesizing of HSPs. The heat shock domain was indicated by the existence of the carboxylic terminal [17]. The molecular weights of HSPs were ranged from 10 to $200 \mathrm{kDa}$. HSPs act as chaperones in their participation in signal induction during heat stress [18]. Furthermore, HSPs divided into 3 clusters based on their molecular weight consisted of high molecular weight (80-114 $\mathrm{kDa})$, HSP70 (69-71 kDa), and low molecular weight (15-30 $\mathrm{kDa})$. HSPs were consisted of six structurally conserved distinct classes including HSP100, HSP90, HSP70, HSP60 (Chaperonins), small HSPs (sHSPs), and ubiquitin (8,5 kDa) [19]. Major HSPs were synthesized in eukaryotes [20].

Based on transcriptome data, heat shock protein (HSPs) family was determined as top seventeen rank of transcription level consisted of $18.1 \mathrm{kDa}$ class I heat shock protein (HSP18.1), $18.1 \mathrm{kDa}$ class I heat shock protein-like, $16.9 \mathrm{kDa}$ heat shock protein $2,18.1 \mathrm{kDa}$ class I heat shock protein, small heat shock protein chloroplastic-like (Figure 3).

\section{Gene Expression}

According to transcriptomics data, 64 DEGs were obtained consisted of 17 up-regulated and 47 down-regulated. Interestingly, eight $(12.5 \%)$ out of 64 genes were belongs to heat shock protein (HSP) family. While the highest transcript was uncharacterized LOC105045046 gene, (Figure 3). The function of this gene in response to heat stress not well characterized.

The expression level of uncharacterized LOC105045046 at 14 DAT was validated using RT-qPCR (Figure 4). This gene was upregulated in leaf and root of progeny $\mathrm{A}$, and down regulated in root of progeny $\mathrm{B}$. This indicated that heat stress is affected of oil palm gene expression profiles. In another study, five genes consisted of OsGSK1, TT1, HSP70-OsEnS45, OsHSP74.8 and OsHSP70 were shown differential expression between rice heat tolerant genotypes Nagina 22 (N22) and Dular [21]. 


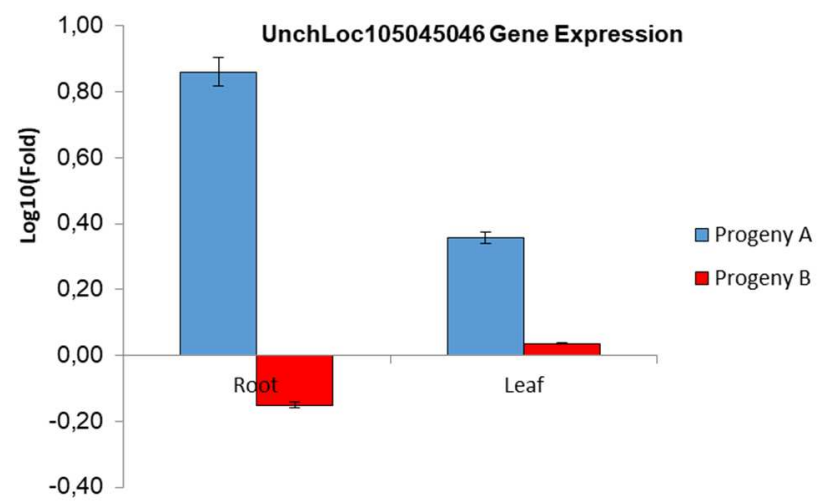

Fig. 4 Uncharacterized LOC105045046 gene expression at 14 DAT

Transcription's level of gene-related heat stress was varied. Fifty percent $(50 \%)$ of the 17 up-regulated genes were the member of HSP gene family. Extreme temperatures can trigger some defense mechanisms. All of the plant organs were reacted to heat stress on the molecular level [22]. HSPs plays for the regulation of the changes during heat stress condition [23], such as enhancing of synthesis especially unexpected changes in genotypic expression.

The general function of HSP100 gene family is in protein aggregation and protein denaturation protection [24]. HSP 90 family consists of proteins that have a highly conserved amino acid sequence. The main functions of HSP90 are signaling transduction networks, cell cycle control, trafficking, control protein folding, and protein degradation. HSP70 families are intensely induced by heat shock and cellular stress, highly maintained through evolution, and constitutively expressed. HSPs70s have important roles under un-stressful conditions. Protein folding and assembly of the subunit is assisting by the highly conserved of approximately $60 \mathrm{kDa}$ protein of HSP60 and chaperons. The small HSPs (sHSPs) family is the most abundant group. The abundance and heterogeneity of sHSPs probably have specific physiological functions. Ubiquitin (8.5 $\mathrm{kDa}$ ) is low molecular weight protein, highly conserved, and located in eukaryotic cells [25].

All 17 top-rank HSPs genes are a member of low molecular weight (LMW-HSP, 15-30 kDa) or small HSPs. The small HSPs are split into six classes based on their cellular compartmentalization, sequence alignments, and immunological cross-reactivity [19]. Class I, II, and III are localized in the cytoplasm or the nucleus, while the other three (IV, V, VI) are located in the chloroplast, the endoplasmic reticulum, and the mitochondria [26]. Three of 18.1 HSPs genes are a member of class I and localized in the cytoplasm. A 16.9 HSP is a member of class II and is localized in the cytoplasm. A small heat shock protein chloroplast-like is including in class IV and localized in the chloroplast.

The other genes contribute to heat sub-categories consisting of BAG-6 gene, chlp-sHSP gene, PHOT-2 gene, and RBOHC gene. The BAG family was a chaperone that regulates diverse cellular pathways consist of programmed cell death and stress response. This gene was controlled by BAG family molecular chaperon regulator protein [27].

The chloroplast sHsp (chlpsHsp) specifically acts as a Photosystem II (PSII) electron transport protector and oxygen evolution during heat stress [28]. An isoform of the smallest chlpsHsp is localized in the thylakoid lumen. They are related to the oxygen-evolving complex (OEC) during heat stress [29]. The activity of PSII is protected from heat stress damage by the chlpsHsp. They did not reactivate heat-denatured PSII [30]. The chlpsHsp gene could be accumulated in high light under non-heat stress conditions [31].

Furthermore, phototropin was blue light receptors that control some responses to optimize plant photosynthetic efficiency. In higher plants, phototropin (phots) are mediated by chloroplast movements and control other light acclimation responses [32]. Phototropins contains two lights, oxygen and voltage (LOV) domains related to PER-ARNT-SIM (PAS) domains. They were presented at the $\mathrm{N}$ - and C-terminal of each phototropin [33]. Plants have two phototropins consist of PHOT1 and PHOT2. The functions of them are overlapping.

Reactive oxygen species (ROS) was identified as signal transduction role in cells. ROS involved in the responses to environmental stimuli, development and cell death, and regulation of growth. Respiratory burst oxidase homologs (RBOHs) were identified as signaling nodes in the ROS gene network of plants. RBOH was integrating a multitude of signal transduction pathways with ROS signaling [5]. ROS was the ability to integrate protein phosphorylation and calcium signaling. Their coupled involvement in a lot of biological processes. RBOHs are located at the cells' ROS network center. RBOH activity could be elevated by abiotic stress stimuli (heat, cold, nutrition, salinity), wounding, and pathogens [36]. Heat stress could induce the activity of the $\mathrm{RBOH}$ gene and elevated the level of expression of this gene in Arabidopsis thaliana [34].

Heavy metal-associated isoprenylated plant proteins (HIPP), slr0575, CML14, and PARP were also identified as the top seventeen up-regulated genes during heat stress on oil palms. (HIPPs) has an important role in plant response to environmental changes such as extreme temperature [27]. Metallochaperone is a key protein for metallic ions transport in the cell [35]. The relative expression of HIPPs was elevated in response to heat stress on oil palm. Slr0575 gene has roles in regulating the expression of thylakoid membrane protein. The thylakoid membrane is the location of the conversion of light to chemical energy by the photosynthesis process. Chlorophyll was located in the thylakoid membrane. Heat stress stimulates changes in photosynthesis and respiration [36]. However, increasing temperature will change in the thylakoid membrane. In this study, Slr0575 gene has relative expression of the 35.51-fold increase in response to high temperature.

Furthermore, a CML14 gene has a role for the regulation of calmodulin-like proteins. These proteins do not have catalytic activity. They act as sensor relays that regulate downstream targets. Several proteins with $\mathrm{Ca} 2+$ binding domains have evolved, such as calmodulin (CaM) and calmodulin-like proteins (CMLs) [37]. These ions were participated in environmental response and development processes in plants. PARP has an important function in many biological processes including transcription, epigenetic regulation, and DNA repair. PARP family expression was 12.15 fold increase response to high temperatures and is linked to abiotic stress response including heat, drought, salinity in Arabidopsis thaliana [38]. 


\section{CONCLUSION}

RNA sequencing analyzed was identified 1,087 genes involved in oil palm heat stress. Sixty-four (64) of them were differentially expressed, consisted of seventeen (17) upregulated and forty-seven (47) down-regulated. Interestingly, $12.5 \%$ out of 64 genes belonged to heat shock protein (HSP) family genes. Uncharacterized LOC LOC105045046 expression level was validated with qPCR. This gene was highly expressed in the leaf of both progenies tested and down expressed in the root of progeny B. The functions and roles of This gene in oil palm heat stress need further characterization together with other differentially expressed genes.

\section{NOMENCLATURE}

cDNA complementary-deoxyribonucleic acid

DAT day after treatment

DEG differential expressed genes

GO gene ontology

RNA ribonucleic acid

rRNA ribosomal ribonucleic acid

TPM transcript per million

Greek letters

$0 \quad$ degree celcius

\section{ACKNOWLEDGMENT}

The authors are grateful to Victor Aprilyanto, Reno Tryono, Marcelinus Rocky Hatorangan, and Widyartini Made Sudania for helpful suggestions regarding this manuscript review. Also, the authors are obliged to Ranny Hanifah, Elsanda Nabila, Fikih Imam Rahman, and Ainun Najib as a technician who support this study in laboratory and research field.

\section{REFERENCES}

[1] F. G. Draft, "Summary for Policy Makers," IPCC, vol. SR I.5, 2018.

[2] O. T. Faulkner, J. R. Mackie, O. T. Faulkner, and J. R. Mackie, The Oil Palm. 2016.

[3] J. H. Christensen and O. B. Christensen, "A summary of the PRUDENCE model projections of changes in European climate by the end of this century," Clim. Change, vol. 81, no. SUPPL. 1, pp. 7-30, 2007, doi: 10.1007/s10584-006-9210-7.

[4] D. B. Lobell and G. P. Asner, "Climate and Management in U . S . Agricultural Yields,” Science (80-. )., vol. 299, no. February, p. 1032, 2003, doi: 10.1126/science.1078475.

[5] F. K. Choudhury, R. M. Rivero, E. Blumwald, and R. Mittler, "Reactive oxygen species, abiotic stress and stress combination," Plant J., vol. 90, no. 5, pp. 856-867, 2017, doi: 10.1111/tpj.13299.

[6] M. J. Paupière, P. van Haperen, I. Rieu, R. G. F. Visser, Y. M. Tikunov, and A. G. Bovy, "Screening for pollen tolerance to high temperatures in tomato," Euphytica, vol. 213, no. 6, 2017, doi: 10.1007/s10681-0171927-z.

[7] S. Ul Haq et al., "Heat shock proteins: Dynamic biomolecules to counter plant biotic and abiotic stresses," Int. J. Mol. Sci., vol. 20, no. 21, pp. 1-31, 2019, doi: 10.3390/ijms20215321.

[8] G. Frank et al., "Transcriptional profiling of maturing tomato (Solanum lycopersicum L.) microspores reveals the involvement of heat shock proteins, ROS scavengers, hormones, and sugars in the heat stress response," J. Exp. Bot., vol. 60, no. 13, pp. 3891-3908, 2009, doi: $10.1093 / \mathrm{jxb} / \mathrm{erp} 234$.

[9] A. Kozomara and S. Griffiths-Jones, "MiRBase: Annotating high confidence microRNAs using deep sequencing data," Nucleic Acids Res., vol. 42, no. D1, pp. 68-73, 2014, doi: 10.1093/nar/gkt1181.

[10] Z. Gu, R. Eils, and M. Schlesner, "Complex heatmaps reveal patterns and correlations in multidimensional genomic data," Bioinformatics, vol. 32 , no. 18, pp. 2847-2849, 2016, doi: 10.1093/bioinformatics/btw313.
[11] A. Feckler et al., "Quantitative real-time PCR as a promising tool for the detection and quantification of leafassociated fungal species - A proof-ofconcept using Alatospora pulchella," PLoS One, vol. 12, no. 4, pp. 1-11, 2017, doi: 10.1371/journal.pone.0174634.

[12] Applied Biosystems, “Applied Biosystems 7000/7500 fast real Time PCR System,” p. 260, 2010, [Online]. Available: http://tools.thermofisher.com/content/sfs/manuals/4387783c.pdf.

[13] N. L. Bray, H. Pimentel, P. Melsted, and L. Pachter, "Near-optimal probabilistic RNA-seq quantification," Nat. Biotechnol., vol. 34, no. 5, pp. 525-527, 2016, doi: 10.1038/nbt.3519.

[14] F. Tian, D. C. Yang, Y. Q. Meng, J. Jin, and G. Gao, "PlantRegMap: Charting functional regulatory maps in plants," Nucleic Acids Res., vol. 48, no. D1, pp. D1104-D1113, 2020, doi: 10.1093/nar/gkz1020.

[15] M. A. García-Parra, D. F. Roa-Acosta, R. Stechauner-Rohringer, F. G.- Molano, D. Bazile, and N. Plazas-Leguizamón, "Effect of temperature on the growth and development of quinoa plants (Chenopodium quinoa Willd.): A review on a global scale," SYLWAN. English Ed., vol. 165, no. 5, pp. 411-433, 2020, [Online]. Available: http://repositorio.unan.edu.ni/2986/1/5624.pdf.

[16] R. K. Singh, V. Gupta, and M. Prasad, "Plant Molecular Chaperones: Structural Organization and their Roles in Abiotic Stress Tolerance," Mol. Plant Abiotic Stress, pp. 221-239, 2019, [Online]. Available: https://onlinelibrary.wiley.com/doi/abs/10.1002/9781119463665.ch1 2.

[17] A. Banerjee and A. Roychoudhury, Small Heat Shock Proteins: Structural Assembly and Functional Responses Against Heat Stress in Plants. Elsevier Inc., 2018.

[18] R. A. Rigg, O. J. T. Mccarty, and J. E. Aslan, Regulation of Heat Shock Protein Responses, vol. 13, no. May. 2018.

[19] M. H. Abou-Deif, M. A.-S. Rashed, K. M. Khalil, and F. E.-S. Mahmoud, "Proteomic analysis of heat shock proteins in maize (Zea mays L.)," Bull. Natl. Res. Cent., vol. 43, no. 1, 2019, doi: 10.1186/s42269-019-0251-2.

[20] E. N. Yer, M. C. Baloglu, and S. Ayan, "Identification and expression profiling of all Hsp family member genes under salinity stress in different poplar clones," Gene, vol. 678, pp. 324-336, 2018, doi: 10.1016/j.gene.2018.08.049.

[21] M. M. S. Wahab, S. Akkareddy, P. Shanthi, and P. Latha, "Identification of differentially expressed genes under heat stress conditions in rice (Oryza sativa L.)," Mol. Biol. Rep., vol. 47, no. 3, pp. 1935-1948, 2020, doi: 10.1007/s11033-020-05291-z.

[22] M. Estravis-Barcala et al., "Molecular bases of responses to abiotic stress in trees," J. Exp. Bot., vol. 71, no. 13, pp. 3765-3779, 2020, doi: $10.1093 / \mathrm{jxb} / \mathrm{erz} 532$.

[23] H. Zhang et al., "Characterization of the heavy-metal-associated isoprenylated plant protein (Hipp) gene family from triticeae species," Int. J. Mol. Sci., vol. 21, no. 17, pp. 1-19, 2020, doi: 10.3390/ijms21176191.

[24] H. A. S. Alhaithloul, "Impact of combined heat and drought stress on the potential growth responses of the desert grass artemisia Sieberi ALBA: Relation to biochemical and molecular adaptation," Plants, vol. 8, no. 10, 2019, doi: 10.3390/plants8100416.

[25] D. Mishra, S. Shekhar, D. Singh, S. Chakraborty, and N. Chakraborty, "Heat Shock Proteins and Abiotic Stress Tolerance in Plants," no. May, pp. 41-69, 2018, doi: 10.1007/978-3-319-74715-6_3.

[26] C. J. Park and Y. S. Seo, "Heat shock proteins: A review of the molecular chaperones for plant immunity," Plant Pathol. J., vol. 31, no. 4, pp. 323-333, 2015, doi: 10.5423/PPJ.RW.08.2015.0150.

[27] I. ullah khan et al., "Identification of novel rice (Oryza sativa) HPP and HIPP genes tolerant to heavy metal toxicity," Ecotoxicol. Environ. Saf., vol. 175, no. March, pp. 8-18, 2019, doi: 10.1016/j.ecoenv.2019.03.040.

[28] X. Sun, J. Zhu, X. Li, Z. Li, L. Han, and H. Luo, “AsHSP26.8a, a creeping bentgrass small heat shock protein integrates different signaling pathways to modulate plant abiotic stress response," pp. 119, 2019, doi: 10.21203/rs.2.16642/v1

[29] E. R. Waters and E. Vierling, "Plant small heat shock proteins evolutionary and functional diversity," New Phytol., vol. 227, no. 1, pp. 24-37, 2020, doi: 10.1111/nph.16536.

[30] S. Hu, Y. Ding, and C. Zhu, "Sensitivity and Responses of Chloroplasts to Heat Stress in Plants Heat Sensitivity of Photosynthesis," vol. 11, no. April, pp. 1-11, 2020, doi: 10.3389/fpls.2020.00375.

[31] M.-H. Avelange-Macherel, A. Rolland, M.-P. Hinault, D. Tolleter, And, and D. Macherel, "The Mitochondrial Small Heat Shock Protein HSP22 from Pea is a Thermosoluble Chaperone Prone to Co- 
Precipitate with Unfolding Client Proteins," Int. J. Mol. Sci., vol. 21, no. 97, pp. 1-23, 2019.

[32] W. Krzeszowiec, M. Novokreshchenova, and H. Gabryś, "Chloroplasts in C3 grasses move in response to blue-light," Plant Cell Rep., vol. 39, no. 10, pp. 1331-1343, 2020, doi: 10.1007/s00299-02002567-3.

[33] M. Hayashi, S. I. Inoue, Y. Ueno, and T. Kinoshita, "A Raf-like protein kinase BHP mediates blue light-dependent stomatal opening," Sci. Rep., vol. 7, pp. 1-12, 2017, doi: 10.1038/srep45586.

[34] M. Hasanuzzaman et al., "Potassium: A vital regulator of plant responses and tolerance to abiotic stresses," Agronomy, vol. 8, no. 3, 2018, doi: 10.3390/agronomy8030031.

[35] Y. Zhao, J. Wang, J. Chen, X. Zhang, M. Guo, and G. Yu, "A Literature Review of Gene Function Prediction by Modeling Gene
Ontology," Front. Genet., vol. 11, no. April, 2020, doi: 10.3389/fgene.2020.00400.

[36] S. Gao et al., "The tomato WV gene encoding a thioredoxin protein is essential for chloroplast development at low temperature and high light intensity," BMC Plant Biol., vol. 19, no. 1, pp. 1-14, 2019, doi: 10.1186/s12870-019-1829-4.

[37] V. La Verde, P. Dominici, and A. Astegno, "Towards understanding plant calcium signaling through calmodulin-like proteins: A biochemical and structural perspective," Int. J. Mol. Sci., vol. 19, no. 5, pp. 1-18, 2018, doi: 10.3390/ijms19051331.

[38] C. Liu et al., "Poly(ADP-ribose) polymerases regulate cell division and development in Arabidopsis roots," J. Integr. Plant Biol., vol. 59, no. 7, pp. 459-474, 2017, doi: 10.1111/jipb.12530. 